\title{
Ascomicetes (Fungi: Ascomycota) del Parque Estatal Agua Blanca, Macuspana, Tabasco, México
}

\section{Ascomycetes (Fungi: Ascomycota) of the Agua Blanca State Park, Macuspana, Tabasco, Mexico}

\author{
Abisag Antonieta Ávalos Lázarol (10. José Edmundo Rosique Gill (D), Silvia Cappello García’,3 (1) \\ José Luis Villarruel Ordaz² (it)
}

1 Universidad Juárez Autónoma de Tabasco, División Académica de Ciencias Biológicas, Herbario UJAT, Rm 0.5 carretera Villahermosa-Cárdenas entronque a Bosques de Saloya, 86150 Villahermosa, Tabasco, México.

2 Universidad del Mar, Instituto de Genética, Campus Puerto Escondido, km 1.5 carretera Puerto Escondido-Sola Vega, 71980 Puerto Escondido, Oaxaca, México.

3 Autor para la correspondencia: cappellogs@hotmail.com

\section{Citar como:}

Ávalos Lázaro, A. A., J. E. Rosique Gil, S. Cappello García y J. L. Villaruel Ordaz. 2018. Ascomicetes (Fungi: Ascomycota) del Parque Estatal Agua Blanca, Macuspana, Tabasco, México. Acta Botanica Mexicana 122: 141-154. DOl: http://dx.doi. org/10.21829/abml22.2018.1261

Recibido: 26 de junio de 2017.

Revisado: 30 de agosto de 2017

Aceptado: 4 de octubre de 2017

Primero en línea: 24 de octubre de 2017.

Publicado: 1 de enero de 2018.

DOI:

http://dx.doi.org/10.21829/abml22.2018.1261

\section{Resumen:}

Antecedentes y Objetivos: La diversidad de los ascomicetos macroscópicos que se conoce actualmente en el Parque Estatal Agua Blanca, Tabasco, es el resultado de varias investigaciones previas sobre todos los macromicetos a nivel estatal. Como no existe un estudio específico sobre los ascomicetos del parque antes mencionado, en este trabajo se reunieron los registros anteriores con los resultados de nuevas exploraciones de este proyecto.

Métodos: El Parque Estatal Agua Blanca se ubica en la región Oeste y Noreste del estado de Tabasco, México. Se realizaron 28 recorridos entre diciembre del 2011 y julio del 2015. El material recolectado se depositó en el herbario UJAT para su preservación e identificación. La identificación de las muestras se realizó siguiendo las técnicas convencionales de micología.

Resultados clave: Se revisaron 129 especímenes, los cuales permitieron la determinación de 22 especies pertenecientes a dos clases, tres órdenes, cinco familias y ocho géneros. Los géneros Scutellinia y Rosellinia se reportan por primera vez para el estado. El género Xylaria es el más diverso con 10 especies, mientras que los géneros Ophiocordyceps, Scutellinia, Hypoxylon y Rosellinia son los menos diversos con una especie cada uno.

Conclusiones: De las 22 especies determinadas, 12(55\%) fueron nuevas citas para Tabasco y 18(82\%) lo son para la zona de estudio, mientras que $10(45 \%)$ ya se habían encontrado previamente en Tabasco y $4(18 \%)$ en el parque. Finalmente, este estudio muestra un mayor número de especies evaluadas en comparación con lo reportado en trabajos previos.

Palabras clave: bosque tropical, diversidad, lignícola, macromicetos, Xylaria.

\section{ABSTRACT:}

Background and Aims: The diversity of the macroscopic ascomycetes currently known from the Agua Blanca State Park, Tabasco, is the result of several previous investigations of macromycetes at the state level. As there is no specific study on the ascomycetes of the aforementioned park, in this work the previous records were combined with the results of new explorations within this project.

Methods: The Agua Blanca State Park is located in the western and northeastern region of the state of Tabasco, Mexico. Twenty eight explorations were carried out between December 2011 and July 2015. The material collected was deposited in the UJAT herbarium for its preservation and identification. Identification of the samples was carried out following the conventional mycological techniques.

Key results: 129 specimens were revised, allowing for the determination of 22 species belonging to two classes, three orders, five families and eight genera. The genera Scutellinia and Rosellinia are reported for the first time for the state. The genus Xylaria is the most diverse with 10 species, while the genera Ophiocordyceps, Scutellinia, Hypoxylon and Rosellinia are the least diverse with one species each.

Conclusions: Of the 22 species identified, 12(55\%) were new records for Tabasco and 18(22\%) were this for the study area, while $10(45 \%)$ of the species had previously been found in the state and $4(18 \%)$ in the reserve. Finally, this study shows a higher number of species evaluated compared to previous work.

Key words: diversity, lignicolous, macromycetes, tropical rain forest, Xylaria. 


\section{INTRODUCCIÓN}

A nivel mundial, los ascomicetos conforman el grupo de hongos más grande, diverso y ecológicamente importante; representan $60 \%$ de las especies y $72 \%$ de los géneros descritos (Medel et al., 1999; González y Hanlin, 2008; Kirk et al., 2008). Su distribución es cosmopolita y se han encontrado colonizando tanto hábitats continentales como marinos, ya sea como saprobios, parásitos o simbiontes, lo cual les permite participar en interacciones ecológicas importantes para el funcionamiento de los ecosistemas.

En México, se han descrito 664 especies de ascomicetos (Aguirre-Acosta et al., 2014), en todo el país. Chiapas, México, Oaxaca y Veracruz son los estados más estudiados, y se tienen pocos registros de Nayarit, Aguascalientes, Baja California, Colima, Sinaloa, Tabasco y Yucatán (Medel et al., 1999; González y Hanlin, 2008). La vegetación tropical y subtropical de estas últimas entidades es predominante y pudiera existir un mayor número de especies de las reportadas, como se ha constatado en otros trabajos (Villegas y Cifuentes, 1988; Medel et al., 1999; Medel, 2002).

En Tabasco, no existe hasta el momento un estudio concreto sobre este grupo; sin embargo, se han reportado especies de ascomicetos en diversos estudios de macromicetos en general (Cappello-García y Hernández-Trejo, 1990; Medel et al., 1999; López-Bonilla y Cappello-García, 2000; Medel, 2002; Cappello-García et al., 2013).

Actualmente se tiene registro de siete especies de ascomicetos en el Parque Estatal Agua Blanca (PEAB) (Cappello-García et al., 2013) y también de la diversidad de macromicetos clavarioides y hongos anamorfos asociados a hojarasca (García-García et al., 2013; Martínez-Rivera et al., 2014; Ávalos-Lázaro et al., 2016). Por lo anterior, el presente trabajo tiene como objetivo describir la diversidad de los ascomicetos del PEAB, así como servir de base para la generación de futuras investigaciones. Esta publicación constituye el primer estudio enfocado en ascomicetos, tanto del área, como del estado de Tabasco.

\section{MATERIALES Y MÉTODOS}

\section{Área de estudio}

El Parque Estatal Agua Blanca (PEAB) se ubica en la región Oeste y Noreste del estado de Tabasco, en el sureste de México, entre los paralelos $17^{\circ} 35^{\prime}$ y $17^{\circ} 38^{\prime} \mathrm{N}$ y los meridianos $92^{\circ} 25^{\prime}$ y $92^{\circ} 29^{\prime} \mathrm{W}$ (Fig. 1). Se encuentra dentro de la región hidrológica Grijalva-Usumacinta. Fue decretado el 19 de diciembre de 1987 como Área Natural Protegida (ANP) de carácter Estatal (SEMARNAT, 2002). Cuenta con una extensión de 2025 ha, dominada por selva alta perennifolia, en la que se conocen alrededor de 1950 especies de plantas vasculares pertenecientes a 150 familias (Castillo-Acosta, 1995; Sánchez-Pérez et al., 2011). El clima es cálido húmedo Af(m)w”(i) g, con temperaturas que oscilan de 23 a $26{ }^{\circ} \mathrm{C}$ y una precipitación pluvial de 2100 a $3200 \mathrm{~mm}$ al año, con abundantes lluvias en verano. Los suelos son de color oscuro tipo rendzinas, formados sobre roca caliza de alto contenido de nutrientes y de materia orgánica, de profundidad promedio de $20 \mathrm{~cm}$ con litosol éutrico fino (Castillo-Acosta y Zavala, 1996; INEGI, 2009; Zarco-Espinosa, et al., 2010).

\section{Recolecta e identificación de los ejemplares}

Para la colecta y descripción de los cuerpos fructíferos de los ascomicetos, se realizaron 28 recorridos al azar, de cuatro horas, en el periodo comprendido entre diciembre 2011 y julio 2015, en diferentes sitios del área de estudio y se siguieron las técnicas básicas de micología propuestas por Cifuentes et al. (1986) y San Martín y Rogers (1995), para lo cual fue necesario el uso de microscopio (Primo Star, Carl Zeizz, Thornwood, EUA).

Para la determinación de las especies se usaron claves de identificación taxonómica y literatura especializada como las de Denison (1963), San Martín y Rogers (1989), San Martín et al. (1997, 1999), Medel et al. (2006, 2008), Sung et al. (2007), Vasilyeva et al. (2007), Hladki y Romero $(2009,2010)$ y Da Silva-Cruz y Cortez (2015). Todos los ejemplares se encuentran depositados en la Colección Micológica del Herbario UJAT (Tabasco, México). 


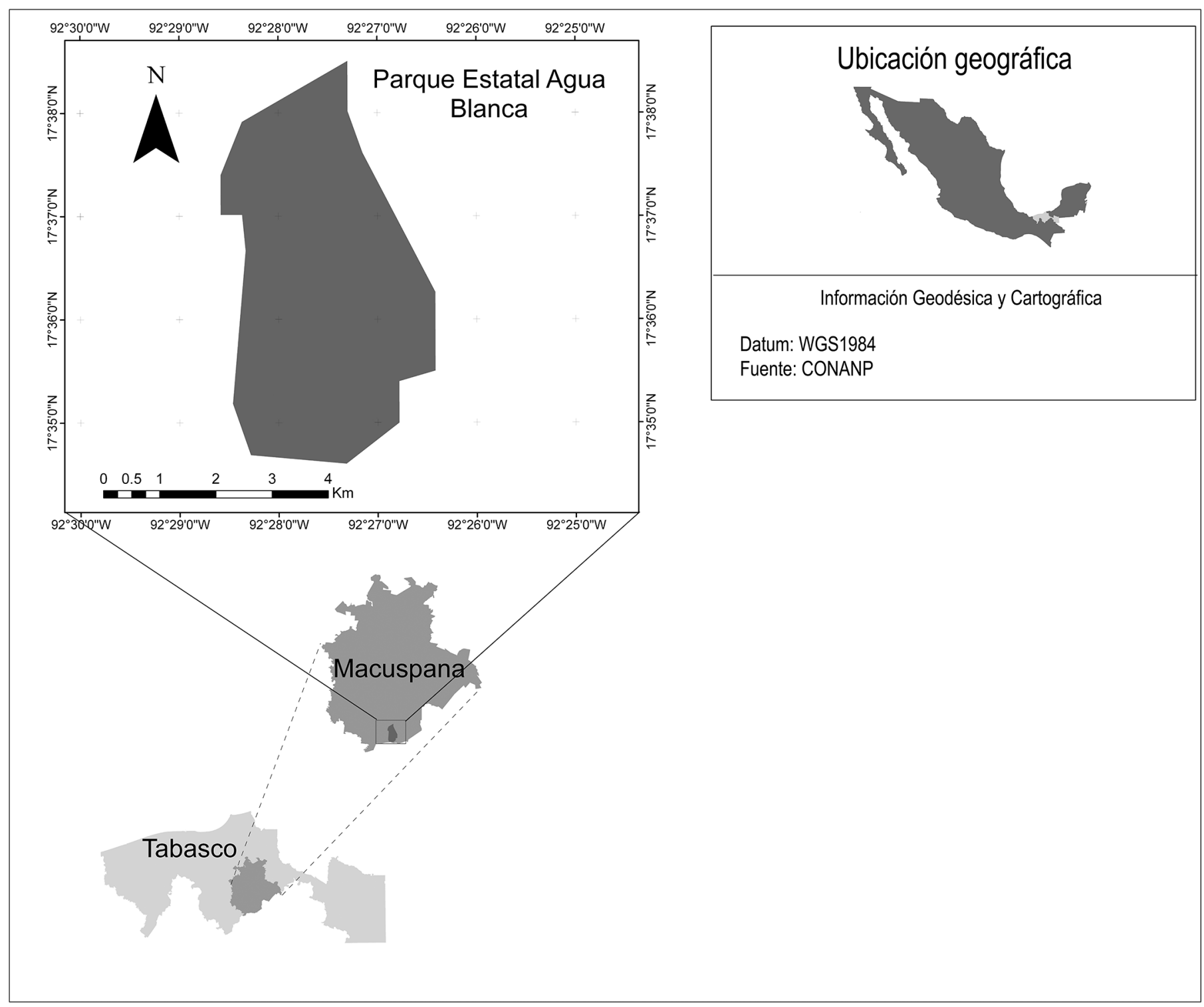

Figura 1: Localización del sitio de estudio en Macuspana, Tabasco, México.

\section{Resultados}

Se estudió un total de 129 especímenes. Taxonómicamente se agruparon en dos clases, tres órdenes, cinco familias, ocho géneros y 22 especies (Cuadro 1). El arreglo taxonómico en el cuadro referido está basado en el Index Fungorum (2017). De los ejemplares identificados, 66(52\%) pertenecen al Orden Xylariales, 55(42\%) a Pezizales y 7(5\%) a Hypocreales. Xylaria Hill ex Schrank fue el género con mayor número de especies (10), mientras que de los géneros Ophiocordyceps Petch, Scutellinia (Cooke) Lambotte, Hypoxylon Adans y Rosellinia De Not. se registró solo una especie.

De las 22 especies estudiadas, 12(55\%) se registran por primera ocasión para el estado, mientras que, para la zona de estudio, 18(82\%) son nuevos registros. Así mismo, los géneros Scutellinia y Rosellinia se registran por primera vez para Tabasco. 
Cuadro 1: Diversidad de familias, géneros y especies de ascomicetos para el Parque Estatal Agua Blanca. Los nuevos reportes para Tabasco están precedidos por un * y para el PEAB por el signo de + .

\begin{tabular}{|c|c|c|}
\hline Familia & Especies & Citas \\
\hline Cordycipitaceae & $\begin{array}{l}\text { Ophiocordyceps sphecocephala (Klotzsch ex Berk.) G.H. Sung, J.M. } \\
\text { Sung, Hywel-Jones \& Spatafora, } 2007\end{array}$ & Cappello et al., 2013 \\
\hline \multirow[t]{6}{*}{ Hypoxylaceae } & +Hypoxylon investiens (Schwein.) M.A. Curtis, 1867 & Medel et al., 1999 \\
\hline & *+Phylacia aff. mexicana Medel, J.D. Rogers \& Guzmán, 2006 & \\
\hline & *+Phylacia globosa Lév, 1845 & \\
\hline & +Phylacia poculiformis (Mont.) Mont., 1885 & Cappello-García y Hernández-Trejo, 1990 \\
\hline & +Phylacia sagrana $(=$ Phylacia sagraeana $)$ Mont., 1856 & Perez-Silva, 1972; Cappello-García y \\
\hline & & Hernández-Trejo, 1990 \\
\hline Pyronemataceae & *+Scutellinia cf. scutellata (L.) Lambotte, 1887 & \\
\hline \multirow[t]{4}{*}{ Sarcoscyphaceae } & +Cookeina speciosa (Fr.) Dennis, 1994 & Cappello-García y Hernández-Trejo, 1990 \\
\hline & Cookeina tricholoma (Mont.) Kuntze, 1891 & Cappello-García et al., 2013 \\
\hline & *+Phillipsia crispata (Berk. \& M.A. Curtis) Le Gal, 1959 & \\
\hline & Phillipsia domingensis Berk., 1981 & Cappello-García et al., 2013 \\
\hline \multirow[t]{11}{*}{ Xylariaceae } & *+Rosellinia corticium (Schwein.) Sacc, 1882 & \\
\hline & *+Xylaria alata F. San Martín \& J.D. Rogers, 1989 & \\
\hline & *+Xylaria aff. filiformis (Alb. \& Schwein.) Fr., 1849 & \\
\hline & *+Xylaria cubensis (Mont.) Fr, 1851 & \\
\hline & + Xylaria guianensis (=guyanensis) (Mont.) Fr., 1851 & Perez-Silva, 1972 \\
\hline & +Xylaria hypoxylon (L.) Grev, 1824 & Cappello-García et al., 2013 \\
\hline & *+Xylaria ianthinovelutina (Mont.) Fr., 1835 & \\
\hline & *+Xylaria mexicana F. San Martín, J.D. Rogers \& P. Lavín, 1998 & \\
\hline & *+Xylaria myosurus Mont., 1855 & \\
\hline & *+Xylaria phyllocharis Mont., 1856 & \\
\hline & Xylaria polymorpha (Pers.) Grev, 1824 & Cappello-García et al., 2013 \\
\hline
\end{tabular}

Las especies Cookeina speciosa (Fr.) Dennis, con 28 especímenes, Cookeina tricholoma (Mont.) Kuntze, con 17 y Phillipsia domingensis Berk. con 10, son las más abundantes dentro del área de muestreo. Phillipsia crispata (Berk. \& M.A. Curtis) Le Gal, Xylaria guianensis (=guayanensis) (Mont.) Fr. (Figs. 2K, L), Hypoxylon investiens (Schwein.) M.A. Curtis y Scutellinia cf. scutellata (L.) Lambotte (Figs. 3G-I; P-U) sólo se registraron una vez. Presentaron hábito lignícola $82 \%$ de las especies, mientras que $9 \%$ se encontró creciendo sobre hojas.

\section{TAXONOMIA}

Nuevos registros para Tabasco

Phillipsia crispata (Berk. \& M.A. Curtis) Le Gal, Bull. Jard. Bot. État Brux. 29: 103. 1959. Fig. 2M.

Apotecios en forma de disco, solitarios a gregarios, sésiles o subsésiles, centralmente adheridos o ligeramente excéntricos, $10-30 \times 2 \mathrm{~mm}$, carnosos y rígidos cuando 

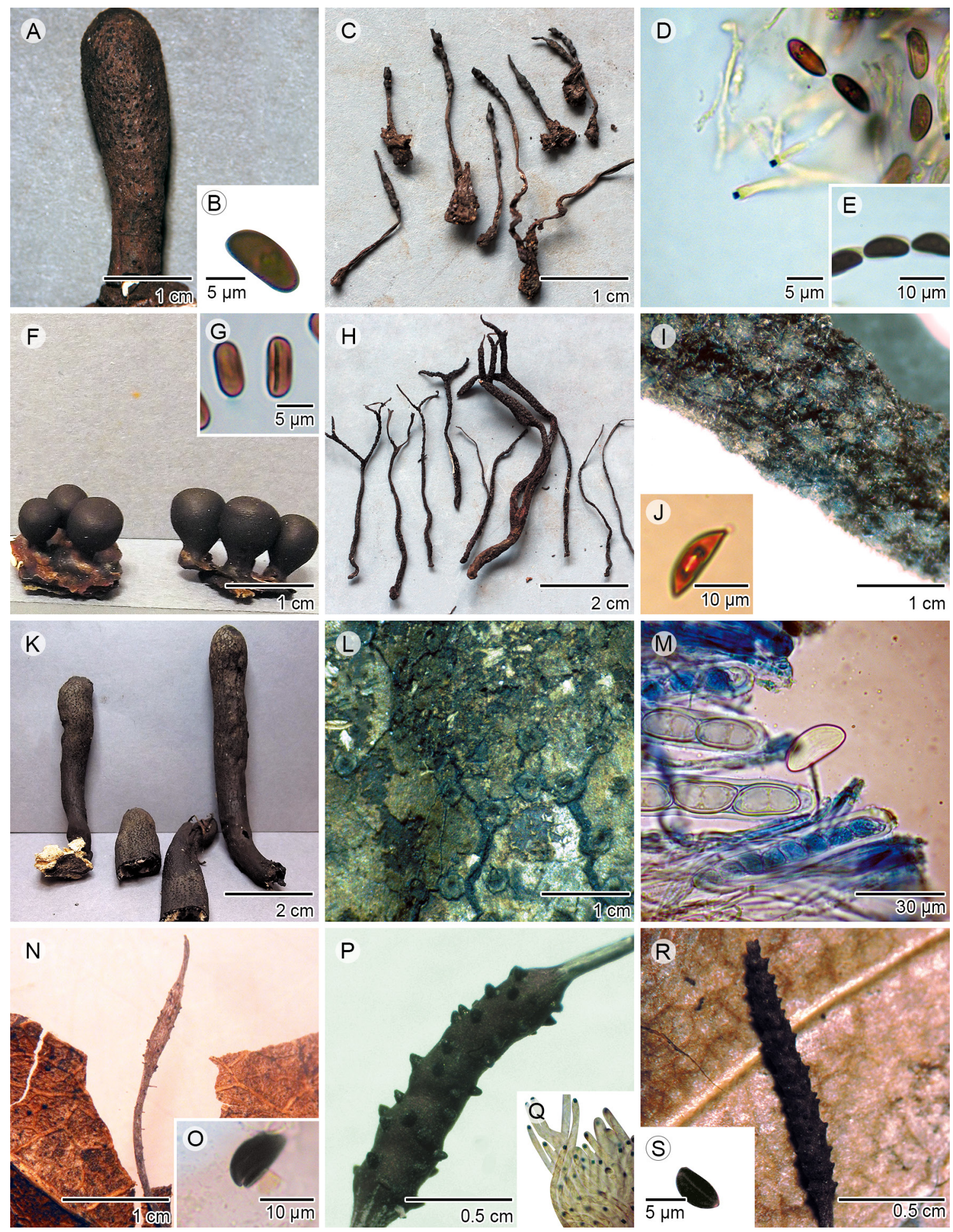

Figura 2: A-B. Xylaria cubensis (Mont.) Fr. (Jiménez-Álvarez 28): A. estroma; B. ascosporas (100x). C-E. Xylaria alata San Martín \& J.D. Rogers (Jiménez-Álvarez 25): C. estroma; D. ascas con aparato apical amiloide en Melzer; E. ascosporas con apéndices alados. F-G. Phylacia mexicana Medel, J.D. Rogers \& Guzmán (Gómez-García 289): F. estroma; G. ascosporas (100x). H-J. Xylaria ianthinovelutina (Mont.) Fr., (Gómez-García 233): H. estroma; I. superficie estromática; J. ascosporas (100x). K-L. Xylaria guianensis (Mont.) Fr., (Jiménez-Álvarez 29): K. estroma; L. superficie estromática; M. Phillipsia crispata (Berk. \& M.A. Curtis) Le Gal, (Sánchez 14): M. ascosporas (100x). N-Q. Xylaria phyllocharis Mont. (Carreño-Ruiz 128): N. estroma; O. ascosporas (100x); P. superficie estromática; Q. ascas con aparato apical amiloide en Melzer (40x). R-S. Xylaria mexicana San Martín, J.D. Rogers \& P. Lavín, (Jiménez-Álvarez 21): R. superficie estromática; S. ascosporas (40x). Fotos por ÁvalosLázaro, A. A. y Gómez-García, V. H. 

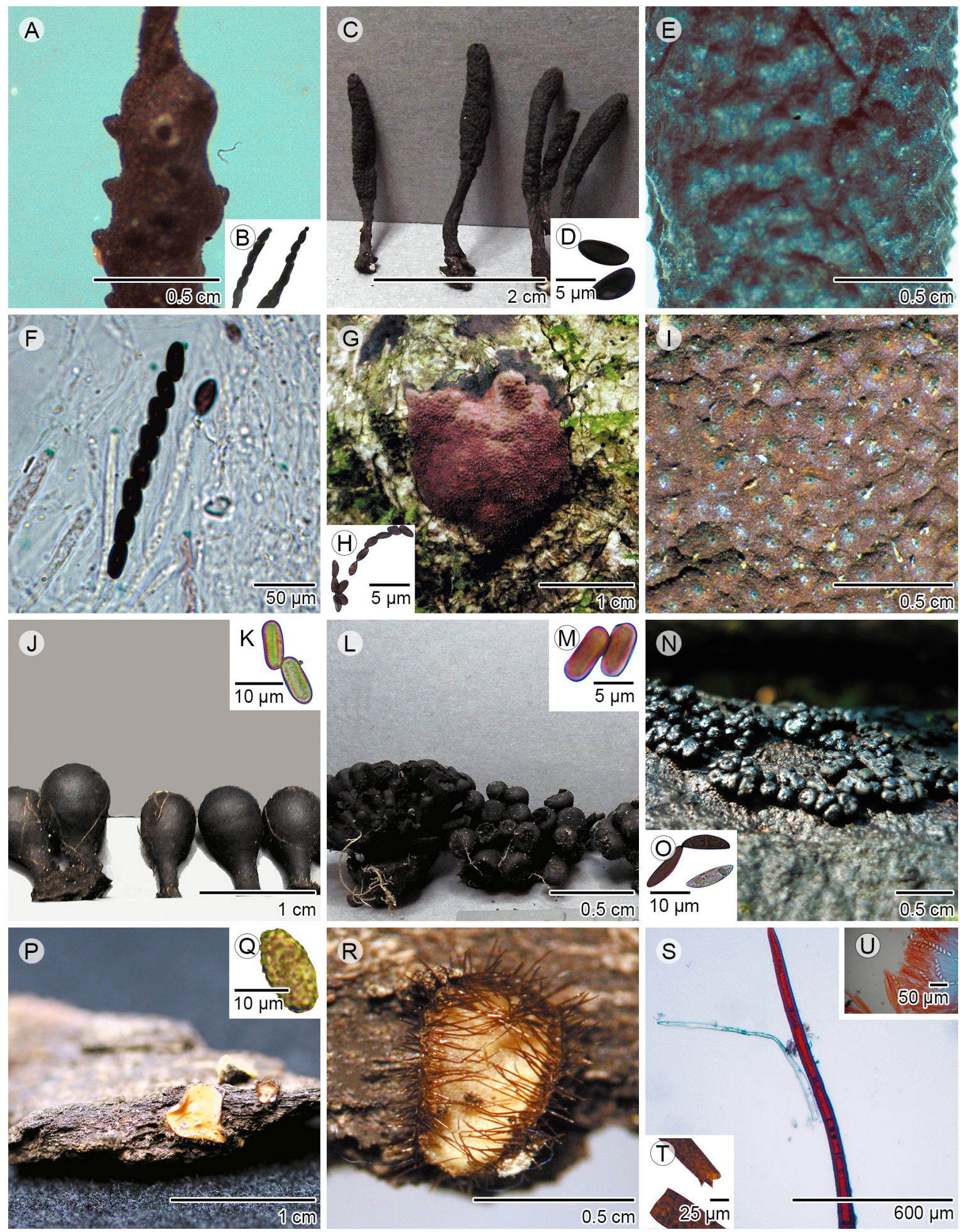

Figura 3: A-B. Xylaria mexicana San Martín, J.D. Rogers \& P. Lavín, (Jiménez-Álvarez 21): A. superficie del estroma; B. aparato apical del asca. C-F. Xylaria myosurus Mont. (Jiménez-Álvarez 51): C. estroma; D. esporas (100x); E. superficie estromática; F. ascas, aparato apical amiloide. G-I. Hypoxylon investiens (Schwein.) M.A. Curtis, (Jiménez-Álvarez 38): G. estroma; H. ascosporas (100x); I. superficie del estroma. J-K. Phylacia globosa Lév, (Mondragón-Sánchez 59): J. estroma; K. ascospora (100x). L-M. Phylacia sagrana Mont. (Gómez-García 527): L. estroma; M. ascosporas (100x). N-O. Rosellinia corticium (Schwein.) Sacc, (Mondragón-Sánchez 60): N. estroma; O. ascosporas (100x). P-U. Scutellinia cf. Scutellata (L.) Lambotte (Gómez-García 652): P. estroma; Q. ascosporas (100x); R. superficie del estroma; S. tricoma; T. base del tricoma; U. ascas operculadas (40x). Fotos por Ávalos Lázaro, A. A y Gómez-García, V. H. 
están frescos, volviéndose ligeramente corchosos cuando secos, color café-morado; ascas con pared delgada, cilíndricas, octosporadas; ascosporas elipsoidales a ligeramente naviculares con bordes agudos, 21-24 × 11-12 $\mu \mathrm{m}$, con más de 10 finas estrías en cada lado; lignícolas.

Distribución: Costa Rica, Cuba y México (Denison, 1969).

Ejemplar examinado: MÉXICO. Tabasco, Macuspana, Parque Estatal Agua Blanca, 28.I.2014, lignícola, Sánchez 14 (UJAT).

Observaciones: esta especie suele ser confundida en campo con $P$. domingensis; sin embargo, el apotecio de $P$. crispata es usualmente de menor tamaño y el color del himenio es distintivamente más café. Microscópicamente son fácilmente diferenciadas por la presencia de estrías en las esporas, que se observan en $P$. domingensis sin necesidad de aceite de inmersión (4-6 estrías por lado), mientras que en $P$. crispata son mucho más delgadas y difíciles de observar (10-14 estrías por lado).

Phylacia globosa Lév, Annls Sci. Nat. Bot., sér. 3, 3: 61. 1845. Figs. 3J, K.

Estromas gregarios, subglobosos, algunas veces piriformes, estipitados, 9-11 mm de diámetro $\times 10-17 \mathrm{~mm}$ de longitud, constreñidos hacia la base formando un estípite 2-3 mm, de solitarios a gregarios, color negro, superficie carbonosa que se desintegra con la edad, endostroma no pudo ser observado en los ejemplares descritos, debido a que solo presentaban el espacio del endostroma sin ningún contenido; peritecios cilíndricos $3 \mathrm{~mm}$ de longitud, en la parte superior del estroma; las ascas no pudieron ser observadas; ascosporas oblongas con línea germinal en el centro color café claro, 9-12 × 4-5 $\mu \mathrm{m}$; lignícolas.

Distribución: Argentina, Brasil, Colombia, Guadalupe, México y Venezuela (Rodrigues y Samuels, 1989; Medel et al., 2006).
Ejemplar examinado: MÉXICO. Tabasco, Macuspana, Parque Estatal Agua Blanca, 19.I.2012, lignícola, Mondragón-Sánchez 59 (UJAT).

Observaciones: Phylacia globosa puede confundirse con P. poculiformis (Mont.) Mont. en campo, pero, difiere de ella por poseer cuerpos subglobosos con ápices redondeados, mientras que $P$. poculiformis se caracteriza por tener forma globosa-piriforme, ligeramente constreñida al centro, con ápices estrechos. El ejemplar analizado posee las esporas ligeramente más delgadas (4-5 vs 5-7.5 $\mu \mathrm{m})$, comparado con lo descrito por Medel et al. (2006).

Phylacia aff. mexicana Medel, J.D. Rogers \& Guzmán, Mycotaxon 97: 282. 2006. Figs. 2F, G.

Estromas gregarios, pseudoturbinados con bordes estromáticos redondeados y ápices planos, 5-9 $\mathrm{mm}$ alto $\times$ 3-6 mm de diámetro, sésiles o estipitados 1-3 × 1-2 mm, color negro, superficie carbonosa, ápices que se desintegran con la edad dejando un orificio por donde son liberadas las esporas; endostroma color blanco, con un punto color negro en el centro del mismo y que se extiende ligeramente hacia el estípite; peritecios cilíndricos hasta 2 $\mathrm{mm}$ de longitud; ascas en forma de saco octosporadas; ascosporas oblongas (7.8-)8-9 × 4-5(-3.7) $\mu \mathrm{m}$, color café amarillento, lignícolas.

Distribución: México (Medel et al., 2006).

Ejemplar examinado: MÉXICO. Tabasco, Macuspana, Parque Estatal Agua Blanca, 23.IV.2012, lignícola, Gómez-García 289 (UJAT).

Observaciones: algunas esporas del material analizado presentan línea germinal que abarca toda la superficie de la espora, contrario a lo mencionado por Medel et al. (2006), quien las describe como carentes de esta línea. Sin embargo, concuerdan con todas las demás características descritas para la especie. Las ascas fueron observadas, pero no pudieron ser fotografiadas para su medición. 
Rosellinia corticium (Schwein.) Sacc., Syll. Fung. (Abellini) 1: 253. 1882. Figs. 3N, O.

Estromas gregarios, redondeados, 1-2 mm de diámetro, uniperiteciados o hasta tres por estroma, rodeados por un subículo color rojizo, que puede estar ausente en organismos muy maduros, superficie más o menos lisa, aplanados en el ápice; con ostiolos papilados; ascas cilíndricas, octosporadas, con aparato apical amiloide en Melzer, 4-5 × 2.4-2.6 $\mu \mathrm{m}$; ascosporas color café rojizo, elipsoidales, algunas de ellas con apéndices hialinos en uno o en ambos lados, lisas, $23-28 \times 6.9-8 \mu \mathrm{m}$ sin tomar en cuenta los apéndices, con línea germinal que casi abarca toda la longitud de la espora, lignícolas.

Distribución: India, norte de Estados Unidos de América y México (Dargan y Thind, 1979; Rogers et al., 2008).

Ejemplar examinado: MÉXICO. Tabasco, Macuspana, Parque Estatal Agua Blanca, 19.I.2012, lignícola, Mondragón-Sánchez 60 (UJAT).

Observaciones: Rosellinia corticium puede ser confundida morfológicamente con $R$. aquila (Fr.) Ces. \& De Not, pero difiere en que ésta posee esporas consistentemente más largas de hasta $31 \mu \mathrm{m}$ y así mismo un aparato apical de mayor tamaño (hasta $11 \mu \mathrm{m}$ ).

Scutellinia cf. scutellata (L.) Lambotte, Mém. Soc. Roy. Sci. Liège, Série 2, 14: 299 (prepr.). 1887. Figs. 3P-U.

Apotecios sésiles, gregarios, color naranja claro, superficie algo curvada, $5 \mathrm{~mm}$ de diámetro; pelos marginales color rojizo alrededor de todo el apotecio, hasta 1 $\mathrm{mm}$ de longitud $\times$ hasta $50 \mu \mathrm{m}$ de ancho, de pared gruesa hasta $8 \mu \mathrm{m}$, con varios septos; ascas cilíndricas octosporadas, 196.4-224 × 10-13.71 $\mu \mathrm{m}$, paráfisis filiformes con ápice clavado 182-252 × 4-6.3 $\mu \mathrm{m}$ de diámetro, ascosporas elipsoidales, $16-20 \times 10-12 \mu \mathrm{m}$, con presencia de gútulas, ornamentadas con verrugas irregulares de más de
$0.5 \mu \mathrm{m}$, algunas aisladas y otras formando placas o crestas, excípulo externo pseudoparenquimático, intricado formado de células globosas de hasta $15 \mu \mathrm{m}$, lignícolas.

Distribución: es una especie de amplia distribución a nivel mundial, en México se ha citado de Chiapas, Ciudad de México, Estado de México, Guanajuato, Hidalgo, Jalisco, Michoacán, Morelos, Nayarit, Oaxaca, Sonora, Tlaxcala y Veracruz (Sierra et al., 2016).

Ejemplar examinado: MÉXICO. Tabasco, Macuspana, Parque Estatal Agua Blanca, 28.I.2014, lignícola, Gómez-García 652 (UJAT).

Observaciones: Scutellinia scutellata podría confundirse macroscópicamente con S. crinita (Bull.) Lambotte; sin embargo, difiere de ésta por la formación de placas a partir de la fusión de la ornamentación de las esporas.

Xylaria alata F. San Martín \& J.D. Rogers, Mycotaxon 34(2): 301. 1989. Figs. 2C-E.

Estromas gregarios, filiformes, simples a ramificados, color negro, delgados, $15-30 \mathrm{~mm}$ longitud $\times$ 0.5-1 $\mathrm{mm}$ de diámetro, parte fértil en el ápice $5-8 \times 1-1.5 \mathrm{~mm}$ de diámetro, cilíndrica con superficie rugosa con apariencia de nudos, por la presencia de peritecios subinmersos, ostiolos papilados; endostroma color blanco a gris; estípite cilíndrico color negro, 10-22 × 0.5-1 mm; ascas octosporadas cilíndricas, (72.94-)75.5-89.2 × 4-5 $\mu \mathrm{m}$, con aparato apical cuadrado, amiloide en Melzer, 1.3-1.7 × 1.3-1.7 $\mu \mathrm{m}$; ascosporas color café, elipsoidesinequilateras, con apéndices hialinos aplanados en cada lado, hialinas, 8.8-10 × 3.7-4 $\mu \mathrm{m}$ incluyendo apéndices, con línea germinal recta que ocupa casi toda la superficie de la espora, en troncos con muy alto nivel de descomposición, lignícolas.

Distribución: México (San Martín y Rogers, 1989; 1995). 
Ejemplar examinado: MÉXICO. Tabasco, Macuspana, Parque Estatal Agua Blanca, lignícola, 29.IX.2012, Jiménez-Álvarez 25 (UJAT).

Observaciones: esta especie se diferencia de otras por la presencia de apéndices aplanados de cada lado de la espora, simulando alas.

Xylaria cubensis (Mont.) Fr. Nova Acta R. Soc. Scient. Upsl., Ser. 3, 1(1): 126. 1851. Figs. 2A, B.

Estromas de solitarios a gregarios, clavados, 18-45 $\mathrm{mm}$ de longitud $\times 4-15 \mathrm{~mm}$ de ancho, ápices redondeados de textura dura pero frágil, todo el estroma color negro a café chocolate, superficie lisa, peritecios totalmente inmersos, diseminados por la superficie del estroma exceptuando la base, con ostiolos papilados; endostroma color blanco y suave, estípite cilíndrico, corto, 4-7 × 2-4 mm; ascas octosporadas, cilíndricas, 150.03-161.93 × 5-6(7) $\mu \mathrm{m}$; con aparato apical cuadrado, amiloide en Melzer, 1.6-1.9 × 1.6-1.9 $\mu \mathrm{m}$; ascosporas color café oscuro a negras elipsoides con las puntas redondeadas, lisas, 7.3-10 $\times$ 4-4.7 $\mu \mathrm{m}$, con línea germinal recta y larga pero difícil de observar claramente; lignícolas.

Distribución: África, América, Asia y Oceanía (Ma et al., 2012; Da Silva-Cruz y Cortez, 2015).

Ejemplares examinados: MÉXICO. Tabasco, Macuspana, Parque Estatal Agua Blanca, lignícola, 28.II.2012, Morales-López 178 (UJAT); loc. cit., 29.IX.2012, Jiménez-Álvarez 23 (UJAT), 28 (UJAT).

Observaciones: Xylaria cubensis es similar a $X$. laevis Lloyd y $X$. allantoidea (Berk.) Fr., pero, difiere de ellas por el tamaño de su aparato apical, en $X$. laevis de menor tamaño $(0.7-1.3 \times 1.2-1.7 \mu \mathrm{m})$ y en $X$. allantoidea es mayor $(2.2-3.6 \times 2-3 \mu \mathrm{m})$.

Xylaria ianthinovelutina (Mont.) Fr. Annls Sci. Nat., Bot., Sér. 2, 3: 339. 1835 Figs. 2H-J.
Estromas gregarios, alargados, delgados, simples o ramificados hacia el ápice, 35-111 $\mathrm{mm}$ de longitud $\times$ 0.5$5 \mathrm{~mm}$ de ancho, en algunos casos hacia el ápice presentan remanentes de su fase asexual, superficie densamente tomentosa, color café oscuro; peritecios subimersos, diseminados por la superficie del estroma exceptuando la base, con ostiolos circulares; endostroma color blanco, con un centro color beige, estípite cilíndrico, tomentoso, $20-50 \times 0.5-5 \mathrm{~mm}$; ascas cilíndricas 88.03-100 × 5-5.5 $\mu \mathrm{m}$ con base larga; con aparato apical, rectangular, amiloide en Melzer, 2-3 × 1-2 $\mu \mathrm{m}$; ascosporas naviculares con bordes ligeramente redondeados, color marrón, 11-13 $\times$ 3.7-4.5 $\mu \mathrm{m}$, línea germinal recta, abarcando la totalidad de la longitud de la espora.

Distribución: Argentina, Brasil, Guyana Francesa, México, Paraguay, Surinam y Trinidad (Dennis, 1956; Da Silva-Cruz y Cortez, 2015).

Ejemplares examinados: MÉXICO. Tabasco, Macuspana, Parque Estatal Agua Blanca, en frutos 24.III.2012, Gómez-García 230 (UJAT), 233 (UJAT), 239(UJAT); 26.VI.2012, Carreño-Ruiz 78 (UJAT); loc. cit. 30.IX.2012, Jiménez-Álvarez 39 (UJAT), 40 (UJAT).

Observaciones: Xylaria ianthinovelutina puede ser confundida con $X$. magnoliae J.D. Rogers, aunque la segunda solo crece en frutos de árboles de la famila Magnoliceae y posee esporas ligeramente de mayor tamaño (10$17 \times 3-6 \mu \mathrm{m}$ ), mientras que la primera es comúnmente encontrada en frutos de la familia Fabaceae. El ejemplar aquí estudiado se encontró creciendo en frutos de Sterculia apetala (Jacq.) H. Karst (Malvaceae).

Xylaria mexicana F. San Martín, J.D. Rogers \& P. Lavín, Rev. Mex. Micol. 13: 66. 1998. Figs. 2R, S; 3A, B.

Estromas solitarios, filiformes inclinados o torcidos sobre la hoja, simples a ligeramente ramificados, 8-25 $\mathrm{mm} \times 1 \mathrm{~mm}$ ancho, color negro, hacia el ápice se encuentra la parte fértil, de forma subglobosa a clavada, 
5-7 $\times 0.5-1 \mathrm{~mm}$, peritecios inmersos con contornos periteciales prominentes, ostiolos hemisféricos sobresalientes; endostroma color blanco, estípite filiforme, corto 4-10× $0.5 \mathrm{~mm}$; ascas cilíndricas octosporadas, (88-)100-120 $\times$ 6-8 $\mu \mathrm{m}$ presentan aparato cuadrado, amiloide en Melzer, 3-4 $\times 3$-4 $\mu \mathrm{m}$; ascosporas color café oscuro elipsoidalesinequilaterales a naviculares, (9.3-)9.5-11.4 × 5.5-6.4 $\mu \mathrm{m}$, con extremos redondeados, con línea central recta que abarca casi la totalidad de la superficie, folícola, sobre la nervadura de las hojas.

Distribución: En México en los estados de Campeche y Chiapas (San Martín et al., 1997).

Ejemplares examinados: MÉXICO. Tabasco, Macuspana, Parque Estatal Agua Blanca, folícola, 29.IX.2012, Jiménez-Álvarez 21 (UJAT); 10.VII.2014, Jiménez-Álvarez 92 (UJAT).

Observaciones: Xylaria mexicana se diferencia de $X$. phyllocharis Mont. por tener las esporas más pequeñas de 9-12 × 5-6 $\mu \mathrm{m}$ vs $12-14 \times 6.5-8.5$, además en $X$. phyllocharis el tomento se restringe solamente al estípite. El estípite $X$. phyllocharis exhiben reminiscencias del anamorfo que van desde bajo de la zona fértil hasta la base del estípite, mientras que en $X$. mexicana las reminiscencias del anamorfo se encuentran en el ápice estéril en forma de estrías.

Xylaria myosurus Mont., Ann. Sci. Nat. Bot. ser. 4, 3: 110. 1855. Figs. 3C-F.

Estromas gregarios, $13-27 \mathrm{~mm}$ alto $\times 1.5-3 \mathrm{~mm}$ ancho, lanceolados o aplanados, color negro, superficie opaca, rugosa con peritecios inmersos, distribuidos por todo el estroma exceptuando la base, ostiolos papilados, endostroma color blanco a beige con el centro color negro, estípite cilíndrico corto, 5-15 × 1.5-2 mm; ascas cilíndricas octosporadas, 81.43-104.3 × 5.13-6.67 $\mu \mathrm{m}$; con aparato apical rectangular, amiloide en Melzer, 2.68-4.38 $\mu \mathrm{m}$; ascosporas 8.2-9.8 $\times$ 4.2-5 $\mu \mathrm{m}$, elipsoida- les-inequiláteras o naviculares, extremos redondeados, línea germinal recta, tenue, ocupando toda la longitud de la espora; lignícolas.

Distribución: Argentina, Brasil, Cuba, GuyanaFrancesa, Nueva Zelanda y México (Dennis, 1956; San Martín y Rogers, 1995 y Hladki y Romero, 2007).

Ejemplares examinados: MÉXICO. Tabasco, Macuspana, Parque Estatal Agua Blanca, 24.III.2012, lignícola, Mondragón-Sánchez 102 (UJAT), 104 (UJAT); loc. cit., 30.IX.2012, Jiménez-Álvarez 43 (UJAT), 51 (UJAT), 52 (UJAT), 70 (UJAT).

Observaciones: el ejemplar aquí estudiado es ligeramente más grande que los descritos por Dennis, (1956) de hasta $20 \times 2 \mathrm{~mm}$ y ascosporas de 7-10 $\times 3-4 \mu \mathrm{m}$. Sin embargo, coinciden en las características descritas para la especie $X$. myosurus en Argentina, por Hladki y Romero (2007), quienes mencionan que el ejemplar tipo estudiado por Dennis se encuentra inmaduro y en malas condiciones; esta especie se caracteriza por sus pequeños cuerpos negros delgados y lanceolados.

Xylaria phyllocharis Mont. Syll. Gen. Sp. Crpt. (Paris): 206. 1856. Figs. 2N-Q.

Estromas solitarios, filiformes, $15-30 \mathrm{~mm}$ longitud $\times$ 0.5-1 mm grosor, parte fértil de ovalada a clavada, color grisáceo, $1-5 \times 0.5-1 \mathrm{~mm}$; peritecios inmersos con presencia de ostiolos prominentes papilados color café a negro; ápice estéril; endostroma color blanco, suave fácil de cortar, estípite filiforme largo, 10-15 $\times 0.5 \mathrm{~mm}$, con presencia de reminiscencias del anamorfo de bajo de la zona fértil, ascas octosporadas cilíndricas, 105-125 × 5-6 $\mu \mathrm{m}$; con aparato apical de cuadrado a rectangular, amiloide en Melzer, 2-4 × 2-3 $\mu \mathrm{m}$; ascosporas color café a café oscuro, elipsoidales con bordes redondeados, lisas, 11.64-13.3 $\times$ 4-5 $\mu \mathrm{m}$, cubiertas con una vaina hialina; línea germinal recta que abarca casi la totalidad de la espora, folícola, sobre las nervaduras de las hojas. 
Distribución: Brasil, Guyana Francesa y México (Dennis, 1956; San Martín y Rogers, 1989 y San Martín et al., 1997).

Ejemplares examinados: MÉXICO. Tabasco, Macuspana, Parque Estatal Agua Blanca, folícola, 24.III.2012, Mondragón-Sánchez 103 (UJAT); loc cit., 24.VI.2012, Mondragón-Sánchez 25 (UJAT), 128 (UJAT), 154 (UJAT); loc. cit., 24.VI.2012, Carreño-Ruiz 128 (UJAT); loc. cit., 25.VI.2012, Mondragón-Sánchez 186 (UJAT).

Observaciones: las esporas de este ejemplar son ligeramente más delgadas de lo que se ha descrito con anterioridad. Quizá se deba a que las esporas se encuentren inmaduras, la mayoría de ellas hialinas dentro de las ascas. Se pudieron encontrar muy pocas esporas de coloración café, cubiertas con una vaina hialina. El interior de los peritecios podía observarse aún de color claro. Xylaria phyllocharis puede confundirse con $X$. asperata J.D. Roges, Rossman \& Samuels, pero se difiere de la misma por tener esporas de mayor tamaño (11-14 vs 9-12 $\mu \mathrm{m})$.

\section{DISCUSIÓN}

El número de especies reportadas en este trabajo representa el mayor registro de ascomicetos tanto para la zona de estudio como para el estado de Tabasco. En listados previos de macromicetos en general solo se citaron alrededor de 12 especies en un mismo artículo (Cappello-García y Hernández-Trejo, 1990; Medel et al., 1999; López-Bonilla y Capello-García, 2000; Medel, 2002). Además, Medel et al. (1999) reportan 20 especies descritas hasta ese momento para la entidad. De las 22 especies aquí reportadas, $45 \%$ (10) ya han sido citadas previamente para el estado, mientras que 18\% (4) habían sido registradas para la zona de estudio (Cappello-García et al., 2013), por lo que se adicionan 18 nuevos registros para el PEAB y 12 para el estado.

Por otra parte, el orden Xylariales fue el más abundante (52\%), lo que concuerda con lo estimado en otras investigaciones, donde se calcula que $75 \%$ de las especies se distribuye en las regiones tropicales del mundo (Whalley, 1993). Así mismo, Medel (2007) y Guzmán (2008) señalan que, hasta 2007, este es el grupo más diverso para México con 335 especies, lo que lo convierten en el mejor conocido para el país.

Los géneros más abundantes, Xylaria y Cookeina Kuntze, concuerdan con los reportes de Medel et al. (1999), Chanona-Gómez et al. (2007), y Medel (2013), quienes encontraron en sus trabajos a Xylaria como el más abundante en los trópicos y en el bosque mesófilo de montaña, sugiriendo que Cookeina puede tener abundancias medias en ambos ambientes.

Chanona-Gómez et al. (2007) han sugerido que la mayoría de hongos lignícolas se distribuyen principalmente en la selva mediana, lo cual concuerda con los resultados obtenidos en el presente estudio. De igual forma, Chanona-Gómez et al. (2007) y Mori del Águila et al. (2011) sostienen que el sustrato más utilizado por los hongos macroscópicos tropicales es la madera de los árboles en descomposición, reportando un mayor número de ejemplares colectados con hábitat lignícola, $63 \%$ y $56 \%$ respectivamente, del total de la muestra.

Las especies Hypoxylon investens (Schwein.) M.A. Curtis, Phylacia sagrana (=Phylacia sagraeana Mont.) (Figs. 3L, M), Xylaria guianensis, X. hypoxylon (L.) Grev, X. polymorpha (Pers.) Grev, Phylacia poculiformis (Mont.) Mont., Cookeina speciosa, C. tricholoma y Phillipsia dominguensis han sido citadas previamente en estudios de macromicetos realizados en el estado (PérezSilva, 1952; Cappello-García y Hernández-Trejo, 1990; Medel et al., 1999; San Martín et al., 1999; López-Bonilla y Cappello-García, 2000; Cappello-García, 2006; Capello-García et al., 2011), mientras que las especies Cookeina tricholoma, Ophiocordyceps sphecocephala (Klotzsch ex Berk.) G.H. Sung, J.M. Sung, Hywel-Jones y Spatafora y Phillipsia dominguensis han sido citadas para el Parque Estatal Agua Blanca (Cappello-García et al., 2013), en el único estudio de macromicetos realizado en la zona.

Es importante señalar que hacen falta investigaciones en este grupo de hongos, tanto en el estado como en las zonas tropicales de México, pues de acuerdo con Guzmán (2008), 
solo se conoce $4 \%$ de los ascomicetos en el país. Por lo tanto, se hace evidente la necesidad de realizar más exploraciones en Tabasco y en otras regiones, que nos permitan seguir documentando y conociendo la diversidad de estos hongos.

\section{CONTRIBUCIONES DE LOS AUTORES}

AAL, JERG, SCG diseñaron la investigación, AAL llevó a cabo el trabajo de campo, las recolectas, la identificación, descripción del material y escribió el texto con ayuda de JERG, SCG y JLVO quienes revisaron el manuscrito y corroboraron las identificaciones y las descripciones taxonómicas. Todos los autores contribuyeron a la discusión, revisión y aprobación del manuscrito final.

\section{FINANCIAMIENTO}

Este estudio fue apoyado por el Consejo Nacional de Ciencia y Tecnología (número de beca 490224 (AAL), y proyecto Tab-2009-C18-122083), y la Universidad Juárez Autónoma de Tabasco (proyecto UJAT-2012-IB-14).

\section{AgradeCIMIENTOS}

Agradecemos a Coral Jazvel Pacheco Figueroa, Rodrigo García Morales y Lilia María Gama Campillo por su valiosa colaboración en la revisión del presente artículo. De igual forma agradecemos a América Mondragón por el apoyo brindado en el trabajo de campo. Así mismo, al laboratorio de ecología del paisaje y cambio global de la Universidad Juárez Autónoma de Tabasco, por la elaboración del mapa del área de estudio y a la diseñadora gráfica Betsabee Elena Ávalos Lázaro por su ayuda en la edición y elaboración de las imágenes.

\section{LITERATURA CITADA}

Aguirre-Acosta, E., M. Ulloa., S. Aguilar., J. Cifuentes y R. Valenzuela. 2014. Biodiversidad de hongos en México. Revista Mexicana de Biodiversidad 85: S76-S81. DOI: https://doi.org/10.7550/rmb.33649

Ávalos-Lázaro, A. A., S. Cappello-García., J. Cifuentes y J. E. Rosique-Gil. 2016. Hongos clavarioides (Agaricomycetes) de Tabasco: diversidad del Parque Estatal Agua Blanca. Revista Mexicana de Micología 43: 19-28.
Cappello-García, S. 2006. Hongos del Yumka': Guía ilustrada. Editorial UJAT. Villahermosa, México. 105 pp.

Cappello-García， S. y H. Hernández-Trejo. 1990. Lista preliminar de los hongos (Macromicetos) y Myxomycetes de Tabasco, México. Universidad y Ciencia 7(13): 15-21.

Cappello-García, S., A. A. Díaz-Contreras y E. LópezHernández. 2011. La diversidad de los hongos de la Sierra. In: López-Hernández, E. (ed.). Educación ambiental para la conservación de la biodiversidad, bases de información para la sierra de Tabasco. Universidad Juárez Autónoma de Tabasco. Villahermosa, México. Pp. 67-87.

Cappello-García, S., E. Rosique-Gil y J. Cifuentes. 2013. Guía de hongos macroscópicos del Parque Estatal Agua Blanca. Universidad Juárez Autónoma de Tabasco. Villahermosa, México. 99 pp.

Castillo-Acosta, O. 1995. Los recursos maderables del estado de Tabasco. Informe técnico. Consejo Nacional de Ciencia y Tecnología. Convenio PC22089. Villahermosa, México. 220 pp.

Castillo-Acosta, O. y C. J. Zavala. 1996. Fisiografía, recursos vegetales y alternativas de manejo en el Parque Estatal Agua Blanca, Tabasco. Universidad y Ciencia 12: 63-70.

Chanona-Gómez, F., R. H. Andrade-Gallegos, J. CastellanosAlbores y J. E. Sánchez. 2007. Macromicetos del Parque Educativo Laguna Bélgica, municipio de Ocozocoautla de Espinosa, Chiapas, México. Revista Mexicana de Biodiversidad 78: 369-381.

Cifuentes, J., M. Villegas y L. Pérez-Ramírez. 1986. Hongos. In: Lot, A. y F. Chiang (eds.). Manual de herbario. Administración y manejo de colecciones, técnicas de recolección y preparación de ejemplares botánicos. Consejo Nacional de la Flora de México, A.C. México, D.F., México. Pp. 55-64.

Dargan, J. S. y K. S. Thind. 1979. Xylariaceae of India: VII. The genus Rosellinia in the Northwest Himalayas. Mycologia 71(5): 1010-1023.

Da Silva-Cruz, K. y V. G. Cortez. 2015. Xylaria (Xylariaceae, Ascomycota) in the Parque Estadual de São Camillo, Paraná Brazil. Acta Biológica Paranaense Curitiba 44(34): 129-144. DOI: http://dx.doi.org/10.5380/abpr.v44i14.43846 
Denison, W. C. 1963. A preliminary study of the operculate cup-fungi of Costa Rica. Revista de Biología Tropical 11: 99-129.

Denison, W. C. 1969. Central American Pezizales. III. The genus Phillipsia. Mycologia 61: 289-304.

Dennis, R. W. G. 1956. Some Xylarias of Tropical America. Kew Bulletin 11(3): 401-444.

García-García, M. A., G. Heredia, S. Cappello-García y E. Rosique-Gil. 2013. Analysis of the sporulating microfungal community in decomposing fallen leaves of Rinorea guatemalensis (Wats.) Bartlett (Malphigiales, Violaceae) in a Mexican Rainforest. Cryptogamie Mycologie 34: 99-111. DOI: http://dx.doi.org/10.7872/ crym.v34.iss 2.2013.99

González, M. C. y R. T. Hanlind. 2008. Distribution and occurrence of Ascomycetes in Mexico. North America Fungi 3(7): 139-145. DOI: http://dx.doi.org/10.2509/ naf2008.003.0077

Guzmán, G. 2008. Análisis de los estudios sobre los macromycetes de México. Revista Mexicana de Micología 28: 7-15.

Hladaki A.I y A. I. Romero. 2007. Primeras citas del género Xylaria (Ascomycota, Xylariaceae) para la república de Argentina. Darwiniana 45(1): 28-44.

Hladki, A. I. y A. I. Romero. 2009. Novedades para los géneros Annulohypoxylon e Hypoxylon (Ascomycota, Xylariaceae) en la República Argentina. Darwiniaina 47: 278-288.

Hladki, A. I. y A. I. Romero. 2010. A preliminary account of Xylaria in Tucuman Province, Argentina, with a key to the known species from the Northern Provinces. Fungal Diversity 42(1): 79-96. DOI: https://doi.org/10.1007/ s13225-009-0008-6

Index Fungorum. 2017. An international project to index all formal names in the Fungi kingdom. http:/www. indexfungorum.org. (consultado marzo de 2017).

INEGI. 2009. Anuario estadístico del Estado de Tabasco México. Instituto Nacional de Estadística, Geografía e Informática. Villahermosa, México.

Kirk, P. M., P. F. Cannon, D. W. Minter y J. A. Stalper. 2008. Ainsworth \& Bisby's Dictionary of the Fungi. 10th ed. CAB International. Wallingford, USA. 784 pp.
López-Bonilla, F. y S. Cappello-García. 2000. Lista de hongos (Macromicetes y Mixomicetes) de la Reserva de la Biosfera Pantanos de Centla. Universidad y Ciencia 15(30): 51-58.

Ma, H. X., Y. Li y L. N. Vasilyeva. 2012. The genus Xylaria in the south of China-5. Three new records in the China Mainland. Österr. Z. Pilzk 21: 61-67.

Martínez-Rivera, K., G. Heredia, E. Rosique-Gil y S. Cappello. 2014. Hongos anamorfos (Hyphomycetes) asociados a restos vegetales de la selva del Parque Estatal Agua Blanca, Macuspana, Tabasco. Acta Botanica Mexicana 107: 99119. DOI: http://dx.doi.org/10.21829/abm107.2014.206

Medel, R. 2002. Nuevos registros de Pyrenomycetes (Ascomycotina) en México. Boletín de la Sociedad Botánica de México 70: 79-85.

Medel, R. 2007. Especies de ascomicetes citados de México IV. 1996-2006. Revista Mexicana de Micología 25: 69-76.

Medel, R. 2013. Hongos ascomicetos del bosque mesófilo de montaña en México. Acta Botanica Mexicana 105: 87106. DOI: http://dx.doi.org/10.21829/abm105.2013.224

Medel, R., G. Guzmán y S. Chacón. 1999. Especies de Macromicetos citadas de México IX. Ascomycetes, parte III: 1983-1996. Acta Botanica Mexicana 46: 57-72. DOI: http://dx.doi.org/10.21829/abm46.1999.816

Medel, R., J. D. Rogers y G. Guzmán. 2006. Phylacia sp nov. and consideration of other species with emphasis on Mexico. Mycotaxon 97: 279-290.

Medel, R., R. Castillo y G. Guzmán. 2008. Las especies de Xylaria (Ascomycota, Xylariaceae) conocidas de Veracruz, México y discusión de nuevos registros. Revista Mexicana de Micología 28: 101-118.

Mori del Águila, T., M. E. Bendayán-Acosta, A. TresierraAyala, M. García-Dávila, E. Ruiz-Sánchez, J. BardalesGarcía, R. Reátegui-Amasifuén, F. Espinoza-Campos y C. Dávila-Flores. 2011. Ascomycetes y Basidiomycetes macroscópicos en bosques de Puerto Almendras (Loreto, Perú). Folia Amazónica 20(1-2): 7-14. DOI: https://doi. org/10.24841/fa.v20i1-2.350

Pérez-Silva, E. 1972. El género Phylacia (Pyrenomycetes) en México. Boletín de la Sociedad Mexicana de Micología 6: 9-15. 
Rodrigues, K. F. y G. J. Samuels. 1989. Studies in the Genus Phylacia (Xylariaceae). Memoirs of the New York Botanical Garden 49: 290-297.

Rogers, J. D., A. N. Miller y L. N. Vasilyeva. 2008. Pyrenomycetes of the Great Smoky Mountains National Park. VI. Kretzschmaria, Nemania, Rosellinia and Xylaria (Xylariaceae). Fungal Diversity 29(9): 107-116.

Sánchez-Pérez, B. R., O. Castillo-Acosta y L. C. CámaraCabrales. 2011. Regeneración natural de la selva alta perennifolia en el parque estatal Agua Blanca, Macuspana, Tabasco, México. Polibotánica 23: 63-88.

San Martín, F. y J. D. Rogers. 1989. A preliminary account of Xylaria of Mexico. Mycotaxon 34: 283-374.

San Martín, F. y J. D. Rogers. 1995. Notas sobre la historia, relaciones de hospedantes y distribución del género Xylaria (Pyrenomycetes, Sphaeriales) en México. Acta Botanica Mexicana 30: 21-40. DOI: http://dx.doi. org/10.21829/abm30.1995.731

San Martín, F., J. D. Rogers y P. Lavín. 1997. Algunas especies de Xylaria (Pyrenomycetes, Sphaeriales) habitantes en hojarasca de bosques mexicanos. Revista Mexicana de Micología 13: 58-69.

San Martín, F., Y. M. Ju y J. D. Rogers. 1999. Algunas especies de Hypoxylon (Pyrenomycetes, Xylariaceae) de México. Acta Botanica Mexicana 47: 31-53. DOI: http://dx.doi. org/10.21829/abm47.1999.823

SEMARNAT. 2002. Áreas naturales protegidas de México con decretos estatales. Secretaria de Medio Ambiente y Recursos Naturales, Instituto Nacional de Ecología,
Consejo Nacional de Áreas Protegidas. Vol 2. México, D.F., México. 907 pp.

Sierra, S., L. Izquierdo-San Agustín, S. Castro-Santiuste, I. Rodríguez-Gutiérrez, A. Alcántara-Mojica, L. PérezRamírez y J. Cifuentes. 2016. Nuevos registros del género Scutellinia (Pyronemataceae, Pezizales) para la micobiota mexicana. Acta Botanica Mexicana 117: 79-89. DOI: http://dx.doi.org/10.21829/abm117.2016.1169

Sung, G. H., N. L. Hywel-Jones, J. M. Sung, J. J. Luangsaard, B. Shrestha y J. W. Spatafora. 2007. Phylogenetic classification of Cordyceps and clavicipitaceous fungi. Studies in Mycology 57: 5-59. DOI: http://dx.doi. org/10.3114/sim.2007.57.01

Vasilyeva, L. N., J. D. Rogers y A. N. Miller. 2007. Pyrenomycetes of the Great Smoky Mountains National Park V. Annulohypoxylon and Hypoxylon (Xylariaceae). Fungal Diversity 27: 231-245.

Villegas, M. y J. Cifuentes. 1988. Revisión de algunas especies del género Ramaria subgénero Lentoramaria en México. Revista Mexicana de Micología 4: 185-200.

Whalley, A. J. S. 1993. Tropical Xylariaceae. Their distribution and ecological characteristic. In: Isaac, S., J. C. Frankland, R. Watling y A. J. S. Whalley (eds.). Aspects of tropical mycology. Cambridge University Press. Cambridge, USA. Pp. 103-120.

Zarco-Espinoza, V. M., J. I. Valdez-Hernández, G. ÁngelesPérez y O. Castillo-Acosta. 2010. Estructura y diversidad de la vegetación arbórea del Parque Estatal Agua Blanca, Macuspana, Tabasco. Universidad y Ciencia 26: 1-17. 